\title{
ON THE GROWTH MECHANISM OF GRAINS IN A PRIMORDIAL STAGE OF THE SOLAR NEBULA
}

\author{
A. CARUSI, A. CORADINI, and C. FEDERICO \\ Istituto di Geologia e Paleontologia, Università di Roma, Italy \\ and \\ M. FULCHIGNONI and G. MAGNI \\ Laboratorio di Astrofisica Spaziale, CNR, Frascati, Italy
}

\begin{abstract}
Grain accretion processes in a protoplanetary nebula have been studied regarding: (a) the distribution function of grain velocities; (b) electrostatic and electromagnetic mechanisms between grains. The velocity distribution function has been investigated for grains embedded in a turbulent gaseous medium. Results have been obtained for protoplanetary nebula densities ranging from $10^{-19}$ to $10^{-10} \mathrm{~g} \mathrm{~cm}^{-3}$. Considering interactions between two grains, photoelectrically charged by galactic ultraviolet flux and by charged-particle capture, and solid-solid interactions (dipole fluctuation effect), the authors estimate the physical cross section $\sigma(v)$ with respect to the geometric one $\sigma_{0}$. Then a statistical approach for an assembly of grains gives the accretion or destruction rates for these small particles. Therefore, according to their characteristic velocities, the following processes have been studied: rupture, fusion, vaporization.
\end{abstract}

\section{Introduction}

The problem of formation of a planetary body may be investigated by studying the accretion processes of dust grains and gas inside a normal interstellar nebula. Many authors have developed this problem, and have obtained analytical solutions for some cases (Cameron, 1972; Safronov, 1972).

Our aim is to investigate grain-accretion processes and to define in some detail the more realistic physical adhesion mechanisms between particles.

The processes examined are:

(a) electrostatic and electromagnetic adhesion;

(b) adhesion by shock-melted material;

(c) destructive mechanisms: rupture or fragmentation by shock melting and vaporization.

These processes are supposed to occur in a turbulent medium: the turbulence may arise in a primordial stage of the nebular collapse (Cameron, 1972).

1. The adopted initial parameter set is consistent with the most recent experimental data (Lequeux, 1972), and also seems to be consistent with the initial theoretical parameters of a collapsing cloud (Larson, 1969, 1972).

For the succeeding calculations, different parameters reflecting the subsequent stages of a collapse have been chosen, in order to evaluate perturbations on the postulated mechanisms of grain growth.

It is difficult to investigate these growth mechanisms in great detail because the chemical composition and the morphological surface structures of grains are poorly 
TABLE I

Set of adopted parameters

$$
\begin{aligned}
& \varrho=10^{-19} \mathrm{~g} \mathrm{~cm}^{-3} \\
& T=10 \mathrm{~K} \\
& g=10^{-10} \mathrm{~cm} \mathrm{~s}^{-2} \\
& Z=1.8 \times 10^{-2} \text { (heavy atoms fraction) }
\end{aligned}
$$

known, as are their physical interactions. Therefore, a homogeneous chemical composition (silica) is adopted for the grains because the cosmical abundances of elements and the interstellar optical extinction seem to agree with a grain chemical composition mixture of silica, graphite, ice, SiC, iron, etc. (Spitzer, 1968; Watson, 1972; Woolf and Ney, 1969; Gillett et al., 1971).

\section{TABLE II}

Grain parameters

$$
\begin{aligned}
& \varrho_{s}=2.3 \mathrm{~g} \mathrm{~cm}^{-3} \\
& \tau_{s}=300 \mathrm{dyn} \mathrm{cm}^{-1} \\
& k_{B}=4 \times 10^{11} \mathrm{~g} \mathrm{~cm}^{-1} \mathrm{~s}^{-2} \\
& Q=6.75 \times 10^{9} \mathrm{dyn} \mathrm{cm}^{-2} \\
& k=1.43 \times 10^{5} \mathrm{erg} \mathrm{s}^{-1} \mathrm{~cm}^{-1} \mathrm{~K}^{-1} \\
& E_{F}=8.5 \times 10^{9} \mathrm{erg} \mathrm{g}^{-1} \\
& E_{V}=5 \times 10^{11} \mathrm{erg} \mathrm{g}^{-1} \\
& T_{F}=1103 \mathrm{~K} \\
& T_{V}=2473 \mathrm{~K} \\
& c_{p}=1.5 \times 10^{7} \mathrm{erg} \mathrm{g}^{-1} \mathrm{~K}^{-1} \\
& \varepsilon_{0}=3.75 \\
& T_{\mathrm{D}}=375 \mathrm{~K} \\
& \chi_{12}=0.1 \\
& \left.\beta_{12}=2 \times 10^{-14} \mathrm{~g} \mathrm{erg}^{-1}\right\}
\end{aligned}
$$

density surface tension bulk modulus stress thermal conductivity melting energy vaporization energy melting temperature vaporization temperature mean specific heat at $p=$ const. static dielectric const. Debye temperature shock wave coefficients

This chemical composition has been chosen because the grains are probably not mainly metallic, owing to the low cosmic abundance of metals in comparison with the mass needed for interstellar extinction (Watson, 1972).

Experimental data show a fluffy and amorphous surface structure caused by cosmic-ray bombardment (Watson, 1972; Greenberg, 1972; Maurette and Bibring, 1972).

In this paper grains are nearly spherical, but corrected by a suitable factor, due to structure roughness.

2. To obtain the different cross sections a Maxwellian distribution of grain velocities has been considered:

$$
f(v)=\frac{4}{\pi^{1 / 2}}\left\langle v^{2}\right\rangle^{3 / 2} \exp \left(-v^{2} \mid\left\langle v^{2}\right\rangle\right),
$$

where $\left\langle v^{2}\right\rangle=\frac{2}{3} w^{2}$, and $w^{2}$ is the variance of the distribution. 
This choice may be justified on the ground of its physical reality and mathematical simplicity (Saffman and Turner, 1956). The physical content of the distribution, however, is summarized in its variance, evaluated in two different ways.

The first model consists of equalating the relaxation length of grains to the size of the smallest eddy, when the relative grain-eddy velocity is comparable to the velocity difference across the smallest eddy (Cameron, 1972; Landau-Lifchitz, 1971).

$$
\left\langle v^{2}\right\rangle_{\mathrm{I}}^{1 / 2}=2.27\left(\varrho_{s} S\right)^{1 / 2}\left(\frac{T}{\varrho}\right)^{1 / 4}
$$

The second model for the variance calculation accounts for the following dynamical effects acting on a grain: turbulence and gravitational field. This theory assumes the following conditions:

(a) grain size smaller than the smallest eddies.

(b) turbulence is isotropic.

The root mean square velocity $\left\langle v^{2}\right\rangle^{1 / 2}$ (deduced by other authors in the past) has been modified, taking into account that when the gas density is low, the drag exerted on the grain is not given by Stokes' law (Cameron, 1972; Epstein, 1924). The time scale of the grain-grain impact phenomenon has been defined by supposing that the relaxation time is equal to the eddies' decay time. In this way it is possible to calculate the length scale of the eddy that governs the impact phenomenon. So the distribution variance is obtained:

where

$$
\begin{aligned}
w_{\mathrm{II}}^{2}=\frac{3}{2}\left\langle v^{2}\right\rangle_{\mathrm{II}}=\frac{1}{3} & \left(s_{1}+s_{2}\right)^{2}{ }_{\eta}^{\varrho \varepsilon}+\left(1-\frac{\varrho}{\varrho_{s}}\right)^{2} \times \\
& \times\left[g^{2}\left(\tau_{1}-\tau_{2}\right)^{2}+4.6 \times 10^{4}\left|\left(\tau_{1}-\tau_{2}\right)\right| T \varrho^{1 / 2}\right],
\end{aligned}
$$

$$
\begin{aligned}
& \tau_{1,2}=\left(\frac{2}{9} \pi s_{1,2}^{2} \varrho_{s}\right) / v_{1,2}^{*} ; \\
& v_{1,2}^{*}=\eta^{*} / \varrho=2.16 \times 10^{3} T^{1 / 2} s_{1,2} \\
& \varepsilon=\text { energy dissipation } \mathrm{g}^{-1} \mathrm{~s}^{-1} \text {. }
\end{aligned}
$$

$\eta^{*}$ is a coefficient derived by Epstein's relationship, $s_{1,2}$ is the radius of the particle, and $\eta=2.38 \times 10^{-6} T^{2 / 3}$ (Cameron, 1972) is the normal Stokes viscosity for the assumed chemical composition.

3. Now some possible physical grain-grain interactions are examined. The first one is related to the possible electric charge of grains. Many authors have considered detailed balance between negative and positive charge, due to the interstellar radiation field and plasma (Feuerbacher et al., 1973; Watson, 1972).

In the UV radiation field the grain potential is calculated by balancing the photoelectron flux and the charge due to bombardment by plasma particles. We assume

$$
F_{0}=1.8 \times 10^{-7} \mathrm{erg} \mathrm{cm}^{-2} \mathrm{~s}^{-1} \AA^{-1} \mathrm{sr}^{-1},
$$

where $F_{0}$ is the UV flux (Hayakawa et al., 1969). 
As regards the charge flux due to ion bombardment, two relationships must be considered.

For negative grain potentials,

$$
\begin{array}{r}
\dot{N}_{\text {stick }}=\frac{2 n_{0} \sigma_{0} a}{\pi^{1 / 2}}\left\{\left(\frac{2 k T}{m_{i}}\right)^{1 / 2}\left[1-\left(\frac{m_{i}}{m_{e}}\right)^{1 / 2} \exp (e V / 300 k T)\right]+\right. \\
\left.-\frac{e V}{300}\left(\frac{2}{k T m_{i}}\right)^{1 / 2}\right\}
\end{array}
$$

and for positive grain potentials,

$$
\begin{aligned}
\dot{N}_{\text {stick }}=\frac{2 n_{0} \sigma_{0} a}{\pi^{1 / 2}}\left\{( \frac { 2 k T } { m _ { i } } ) ^ { 1 / 2 } \left[\exp \left(\frac{-e V}{300 k T}\right)\right.\right. & \left.-\left(\begin{array}{c}
m_{i} \\
m_{e}
\end{array}\right)^{1 / 2}\right]+ \\
& \left.-\frac{e V}{300}\left(\begin{array}{c}
2 \\
k T m_{i}
\end{array}\right)^{1 / 2}\right\} .
\end{aligned}
$$

For the photoelectric effect, the following holds:

where

$$
\dot{N}_{\text {photo }}=\sigma_{0} Y_{0} Q_{0} F_{0} G(V) \text {, }
$$

$$
\begin{cases}G(V)=\frac{1}{\left(E_{0}+V\right)^{2}}-\frac{1}{13.6^{2}} & 0<V<13.6-E_{0} \\ G(V)=\frac{1}{E_{0}^{2}}-\frac{1}{13.6^{2}} & V<0 \\ G(V)=0 & V>13.6-E_{0} .\end{cases}
$$

In Equations (4), we have

$n_{0}=$ concentration of plasma ions

$a=$ capture probability

$\sigma_{0}=$ geometrical cross section

$m_{i}, m_{e}=$ ion and electron masses

$T=$ plasma temperature

$Y_{0}=$ photoemission yield

$Q_{0}=$ photon absorption coefficient

$E_{0}=$ work function

$V=$ potential

Now two grains with radii $s_{1}$ and $s_{2}$, masses $m_{1}$ and $m_{2}$, and electric potential $V_{1}$ and $V_{2}$ attract each other both in the trivial case of oppositely charged grains and in the case of similarly charged grains. The latter occurs when $s_{1} / s_{2} \gg 1$; in fact there is an inductive force produced by the smaller on the bigger particle, able to overcome the normal repulsive effect.

One obtains:

$$
U_{\text {tot }}^{\mathrm{es}}(r)=\frac{V_{1} V_{2}}{r} s_{1} s_{2}-\frac{1 \varepsilon_{0}-1 V_{1}^{2} s_{1}^{2}}{8 \varepsilon_{0}}, r-s_{1},
$$


where $r=$ distance between the centers of grains. The sticking condition between grains is then

$$
\frac{1}{2} \mu v^{2} \leqslant U_{\text {tot }}^{\text {es }}\left(r_{0}\right)-U_{\text {tot }}^{\text {es }}\left(s_{1}+s_{2}\right)=\Delta U_{\text {tot }}^{\text {es }},
$$

where $\mu=m_{1} m_{2} /\left(m_{1}+m_{2}\right)$, and $v$ is the relative velocity.

The maximum value of $U_{\text {tot }}^{\text {es }}(r)$ is found to correspond to $r_{0}$ :

$$
r_{0}=s_{1}\left\{1+\left[2^{3 / 2}\left(\begin{array}{c}
\varepsilon_{0} \\
\varepsilon_{0}-1
\end{array}\right)^{1 / 2} \cdot\left(\frac{V_{2}}{V_{1}}\right)^{1 / 2}\left(\frac{s_{2}}{s_{1}}\right)^{1 / 2}-1\right]^{-1}\right\}
$$

When $r_{0}>s_{1}+s_{2}$, the electromagnetic interaction is attractive (see Figure 1).

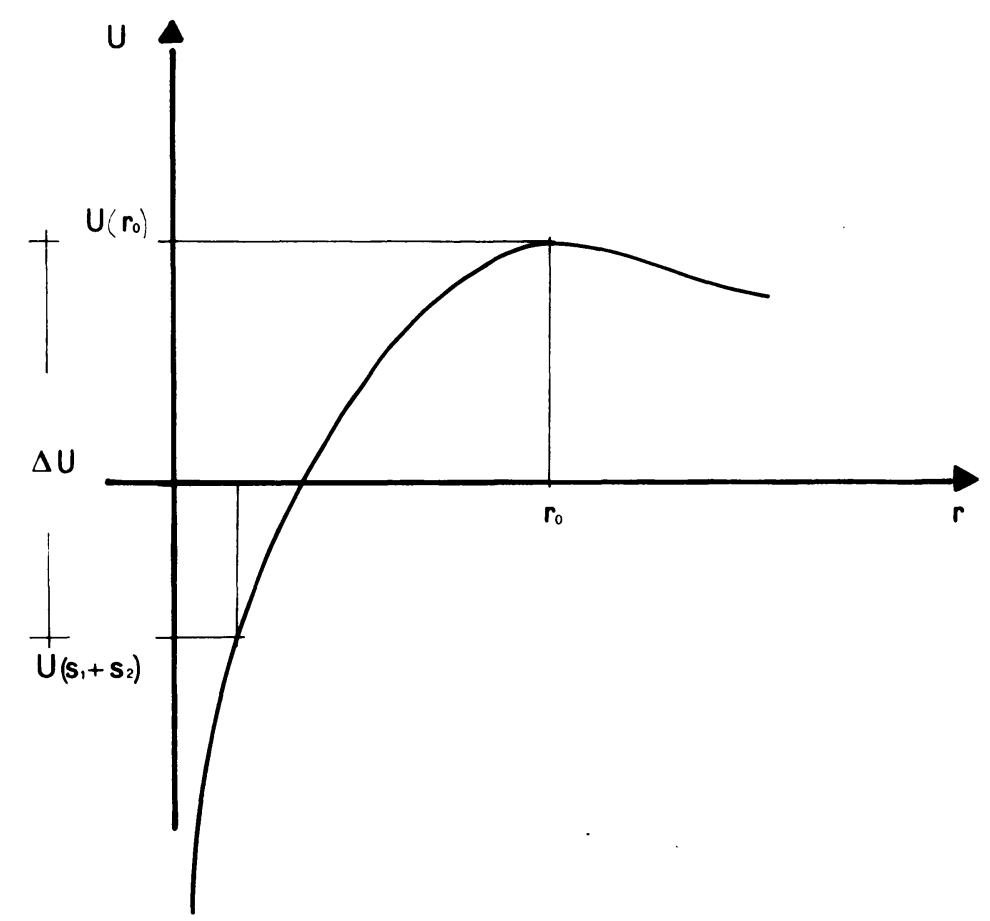

Fig. 1. Electrostatic potential vs. radial distance. In this case the value $r_{0}$ is greater than $s_{1}+s_{2}$, so it is possible to have an attractive potential.

Another effect may be significant when the surfaces of two grains are very close. By applying electromagnetic fluctuation theory, the efficiency of this interaction can be evaluated.

The electromagnetic potential is now critically dependent on the distance: when $r>\lambda_{0}$ (characteristic wavelength of the solid) the attractive potential becomes (for plane surfaces):

$$
U_{\text {tot }}^{\mathrm{em}}(r)=-\frac{A}{r^{3}},
$$


where

$$
\begin{aligned}
& A=\frac{h c \pi^{2}}{720}\left(\begin{array}{l}
\varepsilon_{0}-1 \\
\varepsilon_{0}+1
\end{array}\right)^{2} \varphi\left(\varepsilon_{0}\right) \\
& \varphi\left(\varepsilon_{0}\right) \simeq 0.4 \quad \text { (Landau-Lifchitz, 1969). }
\end{aligned}
$$

In the opposite case $\left(r<\lambda_{0}\right)$, the macroscopic relationship for a spherical grain is (see Appendix)

$$
U_{\mathrm{tot}}^{\mathrm{em}}(r)=-\frac{\hbar}{8 r^{2}} \pi^{2} v_{0} \alpha^{2} n^{2}
$$

where

$$
\begin{aligned}
v_{0} & =\frac{k T_{\mathrm{D}}}{h} \\
\alpha & =\frac{1 \varepsilon_{0}-1}{4 \pi} \frac{n}{n} \\
n & =\text { particle concentration. }
\end{aligned}
$$

In the case of two grains, the total potential becomes (see Appendix):

where

$$
\Delta U_{\text {tot }}^{\text {em }}=3 \pi A P_{4}\left(r_{0}, \lambda_{0}, s_{2}\right)+2 \pi B P_{3}\left(r_{0}, \lambda_{0}, s_{2}\right) \text {, }
$$

$$
A=\frac{2 h c}{720}\left(\frac{\varepsilon_{0}-1}{\varepsilon_{0}+1}\right)^{2} \varphi\left(\varepsilon_{0}\right) ; \quad B=\frac{h \alpha^{2} n^{2} v_{0}}{4}
$$

and $r_{0}=$ minimum approach distance between the grain surfaces.

4. When the two grain strike each other, the collision may be partially inelastic, if the impact velocity is sufficiently high (sound velocity in the grain). Then an amount of energy may be irreversibly trapped.

The interpolation of the results of Carusi et al. (1971) and Coradini (1970) relative to shock coefficients gives the following expression for the amount of trapped energy $\Delta E$ when the target and projectile are siliceous:

$$
\begin{aligned}
\Delta E & =E\left[\alpha\left(m_{1}, m_{2}\right)+\frac{\beta}{m_{2}}\left(m_{1}, m_{2}\right) E\right] \quad\left(m_{1}>m_{2}\right) \\
E & =\frac{1}{2} \mu v^{2} .
\end{aligned}
$$

It is assumed two particles stick when $\Delta E$ is enough to melt at least $10 \%$ of the mass of the smaller one.

That is achieved when:

(a) the radiating time of the trapped heat is less than the characteristic shock time:

$$
\tau_{v}>\tau_{\mathrm{I}}=\gamma \mu E_{F}\left[\frac{\bar{k}\left(\frac{4}{3} \pi s_{2}^{3}\right)^{2 / 3}}{s_{2}}+\sigma_{\mathrm{B}} T_{f}^{4}(4 \pi)^{1 / 3}\left(\frac{\mu}{3 \varrho_{s}}\right)^{2 / 3}\right]^{-1},
$$

where $\sigma_{\mathbf{B}}$ is the Stefan-Boltzmann constant and $\gamma$ is the fraction of molten material. 
It is also possible to obtain another characteristic velocity:

$$
v_{\mathrm{I}}=\frac{\bar{k}\left(\frac{4}{3} \pi s_{2}^{3}\right)^{2 / 3}+\sigma_{\mathrm{B}} T_{f}^{4}(4 \pi)^{1 / 3}\left(\frac{\mu}{3 \varrho_{s}}\right)^{2 / 3}}{\gamma \mu E_{F}}
$$

(b) $\Delta E$ is not enough to vaporize the smallest particle or a fraction $\gamma^{\prime}$ of it (at least $10 \%)$ :

$$
\Delta E<\mu\left[E_{F}+c_{p} T_{V}+\gamma^{\prime} E_{V}\right]
$$

(c) if condition (b) is fulfilled, but not condition (a), the smallest particle completely melts, and adhesion is obtained if the configuration with joined particles is more stable than the disjoined one:

with

$$
E\left(1-\alpha_{12}-\frac{\beta_{12}}{m_{2}} E\right)=\tau_{s}\left(n_{p} S_{n}-S\right)
$$

$n_{p}=$ number of molten fragments, deriving from the original drop;

$S_{n}=$ surface of the $n$th fragment;

$S=$ surface of the original drop.

(d) obviously, the shock must not be able to cause the break-up of the smaller grain. The limit condition results:

$$
E_{R}\left(1-\alpha_{12}-\frac{\beta_{12}}{m_{2}} E_{R}\right)=\frac{\left(Q S \tau_{c}\right)^{2}}{2 \mu}
$$

where $E_{R}$ is the breaking energy, and

$$
\tau_{c}=2 \pi\left[\mu^{2 / 3}\left(\frac{4}{3} \pi \varrho_{s}\right)^{1 / 3} / k_{B}\right]
$$

is the free oscillation time of the solid.

5. It is possible now to define for every mechanism of interaction a characteristic velocity: thus the conditions for constructive and destructive impact may be found.

Electrostatic and electromagnetic interactions cause adhesion between particles when the residual kinetic energy after impact (a fraction of the energy is spent in the shock wave produced) is less than the potential wall produced by these interactions:

$$
E\left(1-\alpha_{12}-\frac{\beta_{12}}{m_{2}} E\right)=\Delta U_{\mathrm{tot}}^{\mathrm{es}}+\Delta U_{\mathrm{tot}}^{\mathrm{em}}
$$

if $\Delta U_{\mathrm{tot}}^{\mathrm{es}}+\Delta U_{\mathrm{tot}}^{\mathrm{em}}>0$, the characteristic velocity $v_{0}$ is obtained.

Moreover, several characteristic velocities are related to the adhesion of grains partially melted by impact (Section 4), which permits one to define the following 
four characteristic velocities:

(a) $\quad E\left(\alpha_{12}+\frac{\beta_{12}}{m_{2}} E\right)=\mu\left(\gamma E_{F}+c_{p} T_{F}\right) \Rightarrow v_{1}$

(b) $\quad E\left(\alpha_{12}+\frac{\beta_{12}}{m_{2}} E\right)=\mu\left(E_{F}+c_{p} T_{V}+\gamma^{\prime} E_{V}\right) \Rightarrow v_{2}$

(c) $\quad E\left(1-\alpha_{12}-\frac{\beta_{12}}{m_{2}} E\right)=\tau_{s}\left(n_{p} S_{n}-S\right) \Rightarrow v_{3}$

(d) $\quad E\left(1-\alpha_{12}-\frac{\beta_{12}}{m_{2}} E\right)=\left(Q n_{p} S \tau_{c}\right)^{2} / 2 \mu \Rightarrow v_{4}$,

where $n_{p}=2$ (breaking of the smaller particle in two equal parts).

TABLE III

Constructive cross-section

\begin{tabular}{lll}
\multicolumn{3}{c}{$\sigma_{c}(v)>0$ for: } \\
$A$ & $B$ & $C$ \\
$v<v_{0}$ & $v>v_{0}$ & $v>v_{0}$ \\
$v<v_{4}$ & $v>v_{1}$ & $v>v_{\mathrm{I}}$ \\
& $v<v_{\mathrm{I}}$ & $v>v_{1}$ \\
& $v<v_{4}$ & $v<v_{2}$ \\
& $v<v_{2}$ & $v<v_{3}$ \\
& & $v<v_{4}$
\end{tabular}

6. The collision of grains, if the impact velocity is great enough, causes their fragmentation. From (14) and (15) it is possible to obtain critical velocities related to fragmentation in the solid (rupture into $n_{p}$ fragments) and liquid states (distribution of matter melted in the shock in $n_{p}$ drops) of grains.

A significant destructive cross section $\sigma_{\mathrm{D}}(v)$ is related to the impact-velocity conditions given in Table IV.

It is thus possible to obtain cross sections for constructive and destructive processes, respectively:

$$
\begin{aligned}
& \sigma_{c}(v)=\sigma_{0} C(v) \\
& \sigma_{\mathrm{D}}(v)=\sigma_{0} D(v),
\end{aligned}
$$

TABLE IV

Destructive cross-section $\sigma_{\mathbf{D}}(v)>0$ for:

\begin{tabular}{ll}
\hline I & II \\
\hline$v 4 \geqslant v_{3}$ & $v_{4}<v_{3}$ \\
$v>v_{3}$ & $v>v_{4}$
\end{tabular}


where

$$
\sigma_{0}=\pi\left(s_{1}+s_{2}\right)^{2}\left[1+\left(\Delta U_{\text {tot }}^{\text {es }}+\Delta U_{\text {tot }}^{\text {em }}\right) / \frac{1}{2} \mu\left\langle v^{2}\right\rangle\right]
$$

is the geometrical cross section corrected for interaction processes between grains, and $D(v)=1$ and $C(v)=1$ if the conditions in Table III and Table IV are satisfied; otherwise $C(v)=D(v)=0$.

The collision frequency for the larger particle is

$$
\dot{v}=N_{g} \int_{0}^{\infty} \sigma(v) v f(v) \mathrm{d} v,
$$

where $N_{g}$ is the number of the smaller grains per $\mathrm{cm}^{3}$.

7. The probabilities of impact between particles have been evaluated for several values of their masses and of the nebular density. A collapsing one solar mass nebula of uniform density has been assumed; cross sections are evaluated at the boundary of the nebula where the gravity is a function of density only.

Results have also been obtained for both models of characteristic turbulent velocity (Section 2).

One can see from the rms turbulent velocity vs nebular density (Figures 2, 3,4) that the general trend of velocity is a decrement with increasing density. The velocity in the first model is, moreover, systematically lower than in the second model, depending on the different characteristic scale length of the turbulence; this effect is particularly evident at low density. The turbulent velocity depends considerably on the mass of the larger particle. In the first model this velocity ranges from 600 to $60 \mathrm{~cm} \mathrm{~s}^{-1}$ (largest-mass grain equal to $1 \mathrm{~g}$ ) and from 60 to $6 \mathrm{~cm} \mathrm{~s}^{-1}$ (largest-mass grain equal to $10^{-12} \mathrm{~g}$ ). For the second model in the same mass ranges the velocities run from $10^{6}$ to $3 \times 10^{3} \mathrm{~cm} \mathrm{~s}^{-1}$ and from $5 \times 10^{3}$ to $60 \mathrm{~cm} \mathrm{~s}^{-1}$.

Figures 2, 3, 4 also illustrate computed values for the frequencies of constructive and destructive collisions, $\dot{\nu}_{c}$ and $\dot{\nu}_{\mathrm{D}}$, between particles of masses $m_{1}$ and $m_{2}$ with $m_{1}>m_{2}$. The cross sections are computed for the larger particles when about 0.015 by mass of the nebular medium is in the smaller grains (Cameron, 1972). The geometrical cross section frequency of collision $\dot{v}_{0}$ is also plotted; so $\dot{v}_{c} / \dot{v}_{0}$ and $\dot{v}_{\mathrm{D}} / \dot{v}_{0}$ show the efficiency of constructive and destructive processes.

The only efficient constructive mechanism is adhesion by interaction between particles, which is governed by velocities $v_{0}$ and $w$ (Sections 2 and 3). For grains of silica, adhesion of molten grains is possible only for extremely low masses; only with a large thermal conductivity (metallic grains) adhesion does become possible in a reasonable mass range (Orowan, 1969). Destructive processes on the contrary are driven by $v_{4}$, because $v_{4}<v_{1}$, and breaking precedes melting in the case of silica.

The characteristic velocity $v_{0}$, if $m_{1} \gg m_{2}$, is essentially driven, for low densities, by electrostatic interaction; while at higher densities photoelectric ejection and ionic capture tend to balance, and electromagnetic interactions become more efficient. For 

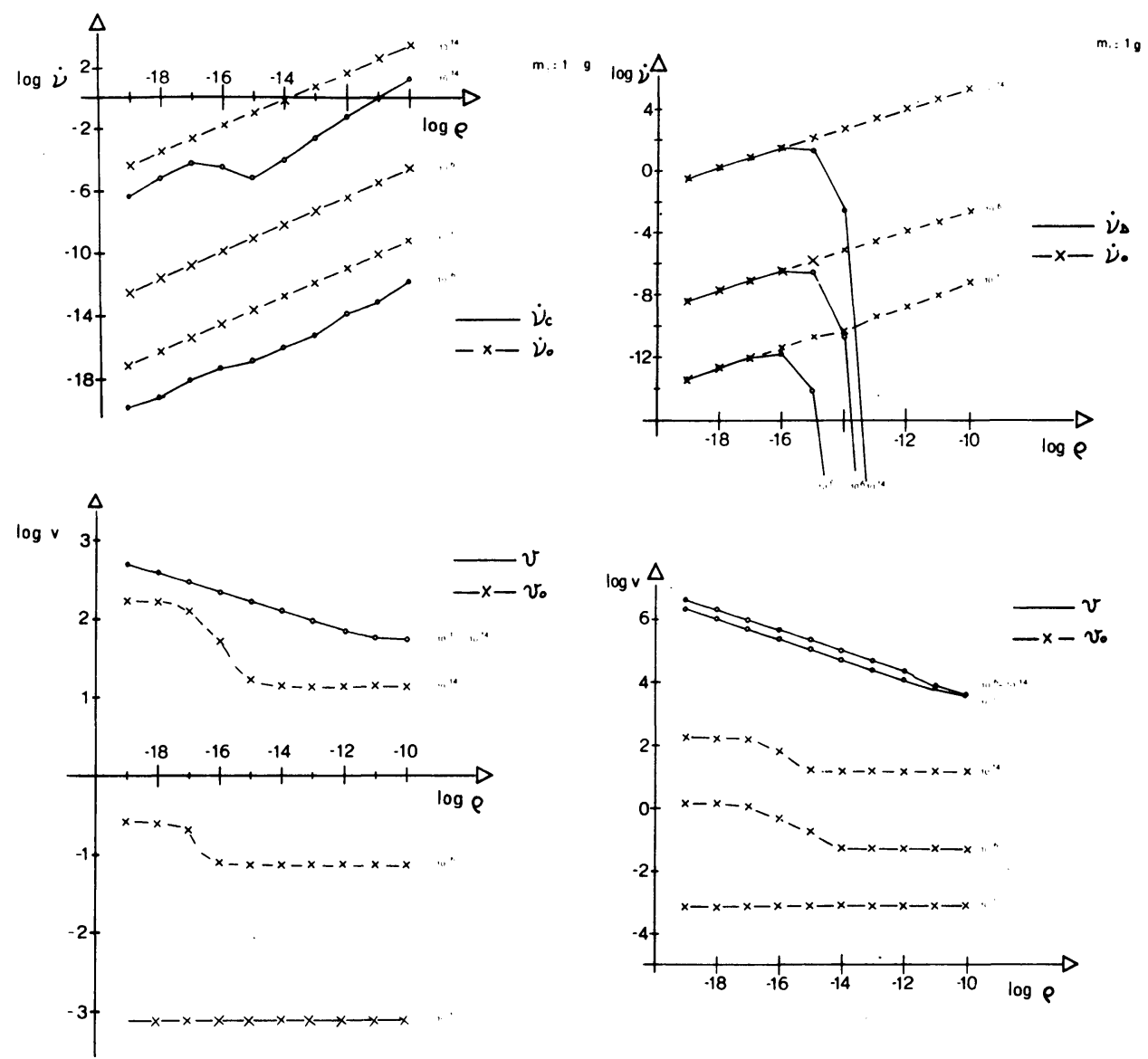

Fig. 2. The left side contains data for model I, the right - for model II. In the upper part is shown the collision frequencies vs nebular density for models I and II, when the mass of the larger grain is $1 \mathrm{~g}$. For model I, the solid line represents the constructive and the dashed line the geometrical collision frequency. For model II, the solid line is the destructive collision frequency and the dashed line is again the geometrical collision frequency. The lower part represents the turbulent and critical velocities in the same conditions: for both models the solid line represents the turbulent velocity for grains of $10^{-1}, 10^{-6}$, and $10^{-14} \mathrm{~g}$, the dashed line represents the characteristic adhesion velocity.

grains of similar masses, the electrostatic interaction is only repulsive; the sole efficient interaction is the electromagnetic one.

A gobal analysis of Figures 2-5 gives some features:

(a) The constructive and destructive processes are essentially governed respectively by the ratios $w / v_{0}$ and $w / v_{4}$; so the collision rates are very different, due to the adopted model for the characteristic velocity of turbulence. Indeed, model I shows constructive collision frequencies higher than model II because of a lower mean impact velocity, while compared with model II it gives small values for destructive collision frequencies.

(b) The collision rates strongly depend on the masses of the colliding particles. In general, a constructive collision is more probable for small colliding particles, or for 

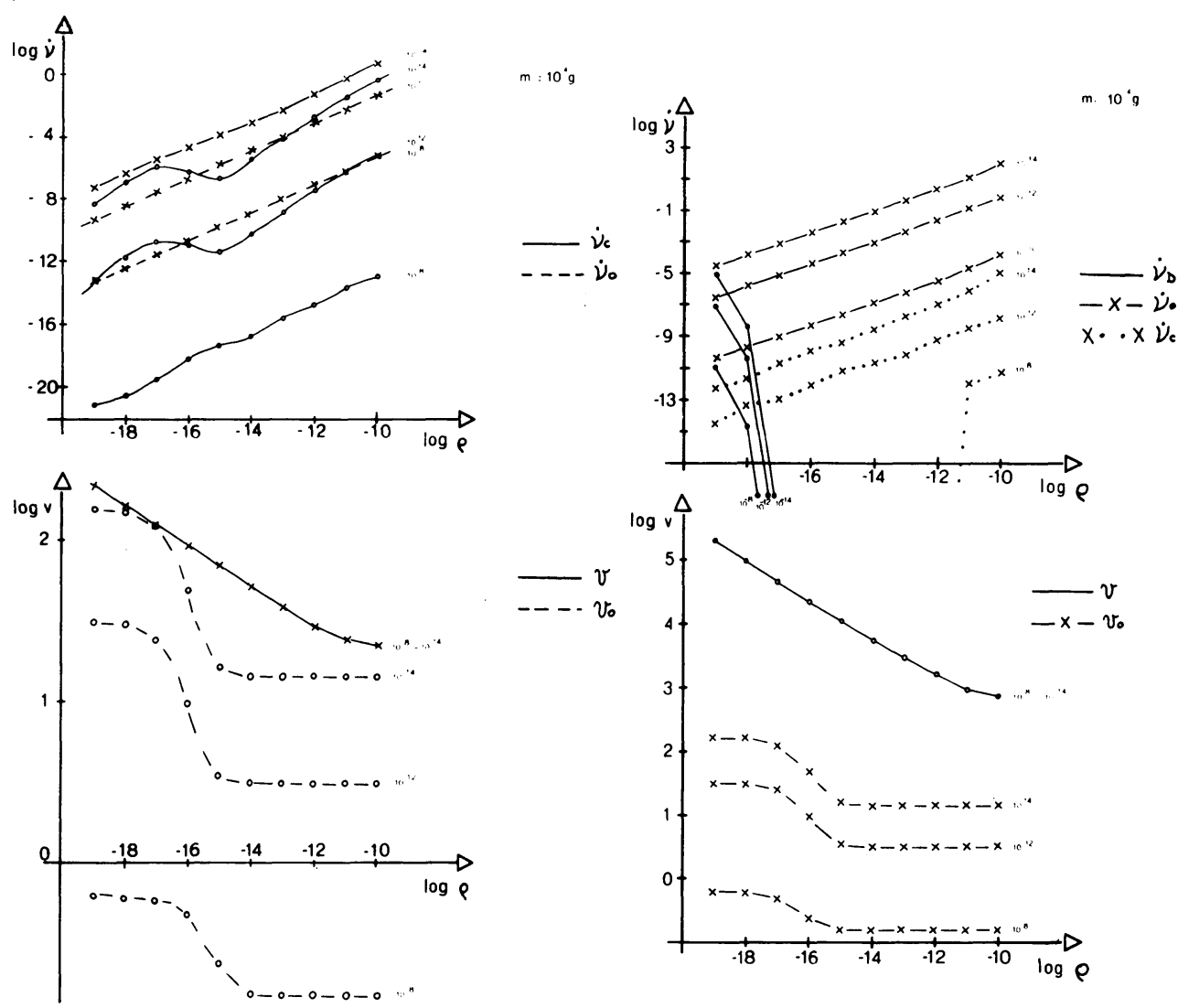

Fig. 3. The left side contains data for model I, the right - for model II. In the upper part is shown the collision frequency vs nebular density for a mass of the larger particle of $10^{-4} \mathrm{~g}$, and masses of impacting particles ranging from $10^{-8}$ to $10^{-14} \mathrm{~g}$. For model $\mathrm{I}$, the solid line represents the constructive collision frequency, the dashed line the geometrical one. For model II the solid line represents the destructive collision frequency, the dashed line the geometrical, and the dotted line the constructive one. In the lower part for the same mass ranges, the turbulent velocity and characteristic adhesion velocity are represented.

particles with a great mass ratio. This is because the mean impact velocity increases with increasing particle mass, and because the interactions considered decrease with increasing mass. In both models, therefore, the frequencies of constructive velocity are negligible for masses of about $1 \mathrm{~g}$.

Efficiency for lower masses can approach $100 \%$ (e.g. the impact of masses of $10^{-12}$ $10^{-13} \mathrm{~g}$ is more efficient than $10 \%$ for $\varrho>10^{-13} \mathrm{~g} \mathrm{~cm}^{-3}$ ).

(c) For model II it is possible to have a destructive interaction that can also reach an efficiency of $100 \%$ if the mass of the larger grain is high enough. The processes' efficiency rapidly decays with increasing density, and becomes less efficient as the particle mass is reduced. It must be taken into account that the collision frequencies refer to the boundary of a one solar mass nebula. Compared to the first model, the 

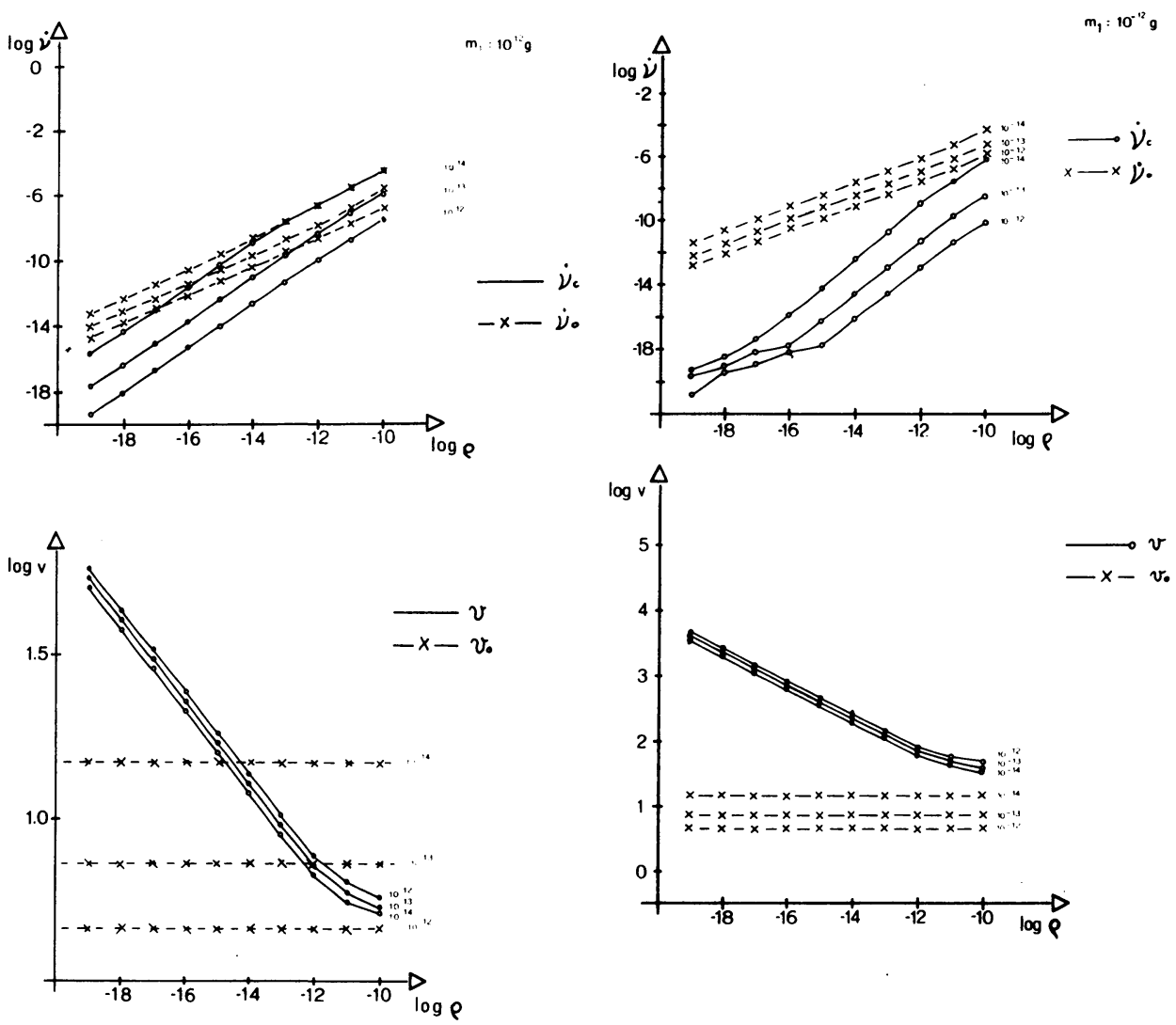

Fig. 4. The left side contains data for model I, the right - for model II. In the upper part is shown the collision frequency vs nebular density for masses of the larger particles of $10^{-12} \mathrm{~g}$, the masses of impacting particles ranging from $10^{-14}$ to $10^{-12} \mathrm{~g}$. For both models the dashed line represents the geometrical frequency distribution, the solid line the constructive one. In the lower part the turbulent velocity (solid line) and characteristic adhesion velocity (dashed line) for the two models are represented.

lower gravity implies a smaller characteristic turbulent velocity and, consequently, a lower constructive-collision efficiency.

\section{Conclusions}

A model based on the adhesion and fragmentation mechanism contains several uncertainties, connected with:

(a) the difficulty of a correct definition of grain interaction processes; and

(b) the uncertainty of physical parameters and the structure and dynamical process of the nebula such as turbulence, radiative balance and opacity.

However, the model can produce many results that, if valid, would indicate that aggregation processes between grains can work on masses less than $10^{-4} \mathrm{~g}$ with sufficient efficiency during several thousand years. 


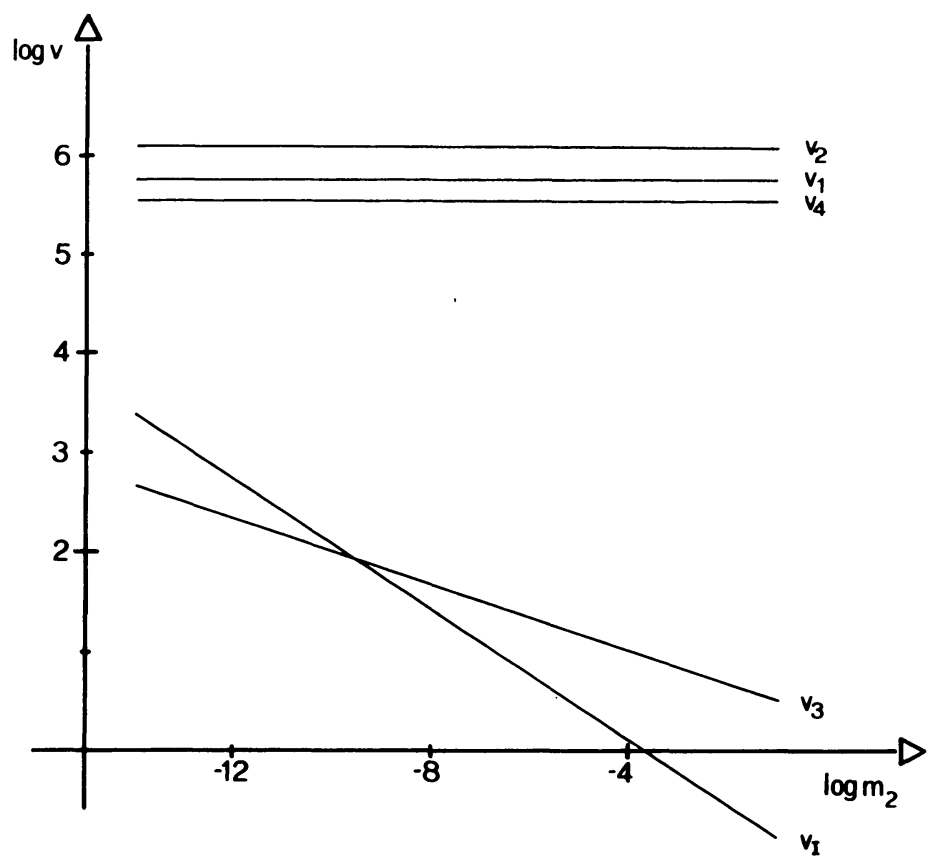

Fig. 5. Critical velocities: $v_{1}, v_{1}, v_{2}, v_{3}$ and $v_{4}$ vs the mass of the smaller considered particles are represented.

A final answer on the probability of grain accretion requires a detailed study of the time evolution of grain masses in a collapsing nebula. The authors are completing a numerical method for defining the time evolution of a fixed-mass distribution function of grains in conditions of gravitational collapse, making use of processes studied in this paper, and also considering accretion of nebular gas captured by grains.

\section{Acknowledgements}

We wish to thank Prof. B. Accordi, who made this work possible; L. Spinozzi, M. Salomone, and O. Fanucci took care of the pictures and graphs.

\section{Appendix}

The electromagnetic interaction between two grains of radii $s_{1}$ and $s_{2}$ may be considered, if $s_{1} \gg s_{2}$, as an interaction between a sphere of radius $s_{2}$ and semispace (Figure 6). The layer of depth $\mathrm{d} r$ contributes to the energy of interaction by

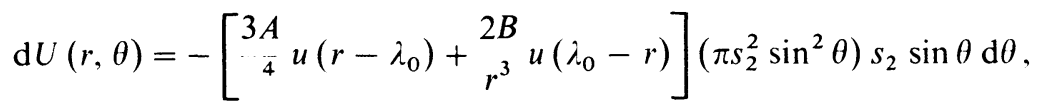




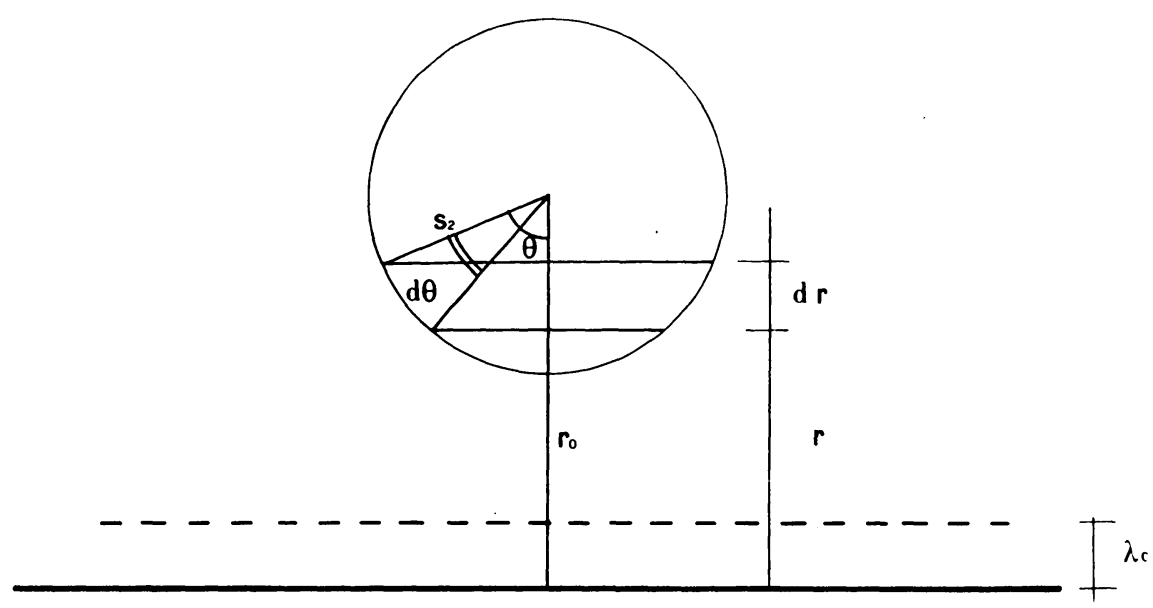

Fig. 6. Illustration of the model considered in the appendix.

where

$$
\begin{array}{ll}
u\left(r-\lambda_{0}\right)=0 & r<\lambda_{0} \\
u\left(r-\lambda_{0}\right)=1 & r \geqslant \lambda_{0} .
\end{array}
$$

The total energy is

$$
U_{\text {tot }}=\int_{0}^{\pi} \mathrm{d} U(r, \theta)=3 A \pi s_{2}^{3} P_{4}\left(r_{0}, \lambda_{0}, s_{2}\right)+2 \pi s_{2}^{3} B P_{3}\left(r_{0}, \lambda_{0}, s_{2}\right),
$$

where if

(1)

$$
\begin{aligned}
\lambda_{0} \leqslant r_{0} \Rightarrow P_{4} & =\int_{0}^{\pi} \frac{\sin ^{3} \theta \mathrm{d} \theta}{\left[r_{0}+s_{2}-s_{2} \cos \theta\right]^{4}}=\int_{0}^{\pi} F_{4}\left(\theta, r_{0}, s_{2}\right) \mathrm{d} \theta \\
P_{3} & =0
\end{aligned}
$$

(2)

$$
\begin{aligned}
& r_{0}<\lambda_{0} \leqslant 2 s_{2}+r_{0} \Rightarrow P_{4}=\int_{\theta_{0}}^{\pi} F_{4}\left(\theta, r_{0}, s_{2}\right) \mathrm{d} \theta \\
& P_{3}=\int_{0}^{\theta_{0}} \frac{\sin ^{3} \theta \mathrm{d} \theta}{\left[r_{0}+s_{2}-s_{2} \cos \theta\right]^{3}}=\int_{0}^{\theta_{0}} F_{3}\left(\theta, r_{0}, s_{2}\right) \mathrm{d} \theta \\
& \theta_{0}=\arccos \left[1+\frac{r_{0}-\lambda_{0}}{s_{2}}\right]
\end{aligned}
$$

(3) $\quad \lambda_{0}>r_{0}+2 s_{2}$

$$
P_{4}=0 ; \quad P_{3}=\int_{0}^{\pi} F_{3}\left(\theta, r_{0}, s_{2}\right) \mathrm{d} \theta
$$

and where the integrals have an easy analytical resolution. 


\section{References}

Cameron, A. G. W.: 1972, Symposium on the Origin of the Solar System, Nice, p. 52.

Carusi, A., Coradini, A., Fulchignoni, M., and Magni, G.:1972, in S. K. Runcorn and H. C. Urey (eds.), 'The Moon', IAU Symp. 47, 180.

Chapman, S. and Cowling, T. G.: 1961, Mathematical Theory of Non Uniform Gases, Cambridge University Press.

Coradini, A.: 1970, Laurea Thesis, University of Roma.

Epstein, P. S. : 1924, Phys. Rev. 23, 710.

Feuerbacher, B., Willis, R. F., and Fitton, B.: 1973, Astrophys.J.181, 101.

Gillet, F. C., Merrill, K. M., and Stein, W. A. : 1972, Astrophys. J. 164, 83.

Greenberg, M. J.: 1972, Symposium on the Origin of the Solar System, Nice, p. 135.

Hayakawa, S., Yamashita, K., and Yoshioka, S. : 1969, Astrophys. Space Sci. 5, 493.

Landau, L. and Lifchitz, E.: 1969, Electrodynamique des milieux continus, MIR.

Landau, L. and Lifchitz, E.: 1971, Mécanique des fluides, MIR.

Larson, B. R. : 1969, Monthly Notices Roy. Astron. Soc. 145, 271.

Larson, B. R.: 1972, Monthly Notices Roy. Astron. Soc. 156, 437.

Larson, B. R.: 1972, Symposium on the Origin of the Solar System, Nice, p. 142.

Lequeux, J.: 1972, Symposium on the Origin of the Solar System, Nice, p. 118.

Maurette, M. and Bibring J. P.: 1972, Symposium on the Origin of the Solar System, Nice, p. 284.

Orowan, E.: 1969, Nature 222, 867.

Saffman, P. G. and Turner J. S.: 1956, J. Fluid. Mech. 1, 16.

Safronov, V. S.: 1972, Symposium on the Origin of the Solar System, Nice, p. 89.

Spitzer, L., Jr. and Tomasko, M. G.: 1968, Astrophys. J. 152, 971.

Watson, W. D.: 1972, Astrophys.J.176, 103.

Wickramasinghe, N. C.: 1967, Interstellar Grains, Chapman \& Hall.

Woolf, N. J. and Ney, E. P.: 1969, Astrophys. J. 155, L181. 